\title{
Multimode Phonon Cooling via Three Wave Parametric Interactions with Optical Fields
}

\author{
G. S. Agarwal \\ Department of Physics, Oklahoma State University, Stillwater, Oklahoma 74078, USA \\ Sudhanshu S. Jha \\ UM-DAE Centre for Excellence in Basic Sciences, \\ University of Mumbai Vidyanagari Campus, Mumbai 400098, India
}

(Dated: October 29, 2018)

\begin{abstract}
We discuss the possible cooling of different phonon modes via three wave mixing interactions of vibrational and optical modes. Since phonon modes exhibit a variety of dispersion relations or frequency spectra with diverse spatial structures, depending on the shape and size of the sample, we formulate our theory in terms of relevant spatial mode functions for the interacting fields in any given geometry. We discuss the possibility of Dicke like collective effects in phonon cooling and present explicit results for simultaneous cooling of two phonon modes via the anti-Stokes up conversions. We show that the bimodal cooling should be observable experimentally.

PACS numbers: 42.50.Wk, 07.10.Cm, 42.65.Es, 42.50.Lc
\end{abstract}

\section{INTRODUCTION}

The cooling of nano mechanical mirrors by the process of optical up-conversion has become a standard technique [1-3]. However the same technique does not quite work for cooling of Brilliouin acoustic modes [4] in a bulk material medium. In this case, one has to satisfy constraints arising from the phase matching conditions and at the same time avoid the generation of Stokes radiation. In the simplest case it is known that the Stokes process dominates [5, 6]. The latter becomes critical because a thermal distribution of phonons would give rise to the generation of both Stokes (down conversion) and anti-Stokes (up conversion) fields. The dominant Stokes process would lead to heating. Bahl et. al. [4] solved this problem by using a sophisticated resonator so that the incident pump and generated anti-Stokes fields were resonant with two different resonator modes, but the generated Stokes field was nonresonant. In addition, they found a suitable Brilliouin mode in the spherical resonator geometry, so that the relevant phase matching condition was satisfied. More generally phonons are known to have many different types of dispersion relations [7, 8] which in turn determine the phase matching conditions and therefore one has to examine the cooling of phonon modes under more general conditions. It is also very interesting to study the cooling of two or even several different phonon modes simultaneously, via anti-Stokes upconversion. This is very relevant in the context of applications in quantum information science when phonons are used as carriers of quantum information. In the light of this, in this paper we develop a more general framework for possible cooling of general phonon modes.

As mentioned above, our main aim here is to present a general theory of laser cooling of low frequency phonon modes in matter, via the anti-Stokes up- conversion process of the inelastic light scattering. Although, our for- mulation will begin with an overview of the known case of laser cooling of a single longitudinal acoustic phonon mode via the anti-Stokes Brillouin scattering process, it will go on to develop a general theory of cooling of low frequency phonon modes of arbitrary polarization, whether these are acoustic or optical phonons, and whether these are modes in an extended bulk matter or in a confined geometry. In fact, the formulation has been kept general enough to be applicable not only to phonons but to any type of low frequency collective excitation modes in matter which can give rise to inelastic light scattering (Raman scattering) via the modulation of the linear optical dielectric function. The cooling via the three -wave anti-Stokes up -conversion is possible in principle for any such mode, provided that it has a sufficiently long life time compared to the resonant cavity anti-Stokes optical mode, and the corresponding Raman tensor coupling of the mode is not too weak. We will also consider the possibility of cooling more than one phonon mode simultaneously via the three-wave up-conversion process, which has not been considered earlier even for the case of longitudinal acoustic phonons [4, 9].

The organization of the paper is as follows-In Sec II we introduce the basic features of the phonon photon interaction, relevant to anti-Stokes generation. We derive the basic equations for the coupled phonon and electromagnetic fields. In Sec III we derive the equations for phonon and photon fields in terms of the relevant resonator modes. The nature of the phonon dispersion or its frequency spectrum, and its relevance for phonon-photon interaction are discussed in Sec IV. In Sec V we describe quantum Langevin equations and show how the single mode cooling emerges [9]. In Sec VI we discuss collective effects in simultaneous cooling of two modes. The numerical results for the simultaneous cooling of two modes are given in Sec VII, with a discussion of these results. 


\section{BASIC EQUATIONS FOR INTERACTION OF ELECTROMAGNETIC FIELDS WITH VIBRATIONAL FIELDS}

In this section, we present, for completeness, a first principle derivation of the basic equations for the Stokes and anti-Stokes scattering from vibrational modes. This basic description would enable us to formulate the problem of more general three wave interactions in a resonator. For simplicity, let us first consider the case of longitudinal acoustic waves in matter, which give rise to the Brillouin scattering. The long wavelength longitudinal acoustic waves can be described either in terms of density fluctuations $\Delta \rho(\mathbf{r}, t)$ or the displacement field $\mathbf{u}(\mathbf{r}, t)$, which are related by

$$
\frac{\Delta \rho(\mathbf{r}, t)}{\rho_{0}}=-\nabla \cdot \mathbf{u}(\mathbf{r}, t)
$$

where $\Delta \rho, \rho_{0}$ are, say, mass densities, and $\rho_{0}$ is the mean mass density of the medium. In terms of the displacement field $\mathbf{u}(\mathbf{r}, t)$, the unperturbed Hamiltonian of the acoustic field is given by

$$
\begin{aligned}
H_{S} & =\int d^{3} \mathbf{r} \mathcal{H}_{S}, \\
\mathcal{H}_{S} & =\frac{1}{2} \rho_{0} \dot{u}^{2}+\frac{1}{2} \rho_{0} v_{S}^{2}(\nabla \cdot \mathbf{u})^{2},
\end{aligned}
$$

where $v_{S}$ is the sound velocity. Also, for longitudinal sound wave, $\rho_{0} v_{S}^{2}=B$, which is the inverse of the compressibility $c_{S}$. The equation (2) leads to the wave equation for sound waves

$$
\frac{\partial^{2} \mathbf{u}}{\partial t^{2}}-v_{S}^{2} \nabla^{2} \mathbf{u}=\frac{\mathbf{f}_{\mathrm{ext}}}{\rho_{0}},
$$

where $\mathbf{f}_{\text {ext }}$ is external force if any. Because of the sound wave, the electromagnetic polarization of the medium is modulated by the density wave. The change $\Delta \mathbf{P}$ in polarization can be written in terms of the dielectric function as

$$
\begin{aligned}
\Delta \mathbf{P} & =\Delta\left(\frac{\epsilon-1}{4 \pi}\right) \mathbf{E} \\
& =\frac{1}{4 \pi}\left(\frac{\partial \epsilon}{\partial \rho}\right)_{0} \Delta \rho \mathbf{E}=\frac{\rho_{0}}{4 \pi}\left(\frac{\partial \epsilon}{\partial \rho}\right)_{0} \frac{\Delta \rho}{\rho_{0}} \mathbf{E} \\
& =-\frac{\gamma_{e}}{4 \pi}(\nabla \cdot \mathbf{u}) \mathbf{E}
\end{aligned}
$$

where

$$
\gamma_{e} \equiv \rho_{0}\left(\frac{\partial \epsilon}{\partial \rho}\right)_{0}
$$

is the so called electrostrictive constant. For simplicity we consider an isotropic medium. The interaction density (interaction between the sound wave and optical fields) is thus given by [10]:

$$
\mathcal{H}_{\text {int }}=-\frac{1}{2} \Delta \mathbf{P} \cdot \mathbf{E}=\frac{\gamma_{e}}{8 \pi}(\nabla \cdot \mathbf{u}) E^{2},
$$

so that the interaction Hamiltonian $H_{\text {int }}=\int \mathcal{H}_{\text {int }}(\mathbf{r}) d^{3} \mathbf{r}$. In view of the volume integration, Eq. (7) also be written as

$$
\mathcal{H}_{\text {int }}=-\frac{\gamma_{e}}{8 \pi}(\mathbf{u} \cdot \nabla) E^{2}
$$

From Eq. (8) we can obtain the force $f_{\alpha}$ acting on the vibrational mode which would be $-\partial \mathcal{H} / \partial u_{\alpha}$, i.e.

$$
\mathbf{f}=\frac{\gamma_{e}}{8 \pi} \nabla E^{2}
$$

The quantity $-\frac{\gamma_{e}}{8 \pi} E^{2}$ can be identified as the pressure due to electromagnetic waves. Using (9) the wave equation for $\mathbf{u}$ become

$$
\frac{\partial^{2} \mathbf{u}}{\partial t^{2}}-v_{S}^{2} \nabla^{2} \mathbf{u}-\Gamma^{\prime} \nabla^{2} \frac{\partial \mathbf{u}}{\partial t}=\frac{\gamma_{e}}{8 \pi \rho_{0}} \nabla E^{2},
$$

where we have also introduced the damping parameter $\Gamma^{\prime}$ for sound waves.

The electromagnetic field $\mathbf{E}$ satisfies wave equation

$$
\nabla^{2} \mathbf{E}-\frac{\epsilon}{c^{2}} \frac{\partial^{2} \mathbf{E}}{\partial t^{2}}=\frac{4 \pi}{c^{2}} \frac{\partial^{2}(\Delta \mathbf{P})}{\partial t^{2}},
$$

where $\epsilon$ is the linear dielectric function of the medium. In writing the above equation, it has to be noted that we have ignored the linear dispersion of the dielectric function (which leads to the corresponding group velocity instead of the phase velocity) and the linear absorption of optical fields. Later we will however add the linear absorption term as a phenomenological parameter.

Using Eq. (5), Eq. (11) reduces to

$$
\nabla^{2} \mathbf{E}-\frac{\epsilon}{c^{2}} \frac{\partial^{2} \mathbf{E}}{\partial t^{2}}=-\frac{\gamma_{e}}{c^{2}} \frac{\partial^{2}}{\partial t^{2}}[(\nabla \cdot \mathbf{u}) \mathbf{E}]
$$

The analysis given above can be generalized to any type of vibrational mode in an arbitrary medium, whether it is an acoustic or optical phonon mode, a longitudinal or transverse mode in a bulk material, or whether it is a mode in a confined geometry. We can expand $\epsilon$ as a Taylor series in the relevant phonon displacement field $\mathbf{Q}(\mathbf{r}, t)$ whence

$$
(\Delta \mathbf{P})_{i}=\sum_{j k} \frac{1}{4 \pi} \frac{\partial \epsilon_{i j}}{\partial Q_{k}} E_{j} Q_{k}=\sum_{j k} R_{i j k} E_{j} Q_{k} .
$$

where $R_{i j k}$ is the generalized Raman tensor for the mode Q. If more than one phonon mode is involved, then Eq. (13) is to be summed over contributions from all the phonon modes. The interaction Hamiltonian density now becomes

$$
\mathcal{H}_{\text {int }}=-\frac{1}{2} \sum_{i j k} R_{i j k} E_{j} E_{i} Q_{k} .
$$

It should be emphasized here that we have introduced a new variable $\mathbf{Q}$ for the phonon displacement field for a general phonon mode in matter, instead of the variable $\mathbf{u}$ 
used for the low frequency longitudinal acoustic displacement field, on purpose. Both have the same dimensions of length. But, whereas $\mathbf{u}$ is related to the density fluctuation via Eq. (11), and $\nabla \cdot \mathbf{u}=0$ for transverse modes, $\mathbf{Q}$ represents the displacement field for any phonon mode, whether it is transverse or longitudinal, or whether it is an acoustic mode or an optical mode. In fact, in the plane-wave representation for the case of bulk matter, for a mode with wave vector $\mathbf{q}$ and a given general dispersion relation $\Omega(\mathbf{q})$, the displacement field $\mathbf{Q}$ satisfies the equation, $\frac{\partial^{2} \mathbf{Q}}{\partial t^{2}}+\Omega^{2}(\mathbf{q}) \mathbf{Q}=0$, with its velocity $\mathbf{v}=\partial \Omega / \partial \mathbf{q}$. For the low frequency longitudinal acoustic mode, $\mathbf{Q}$ will be the same as $\mathbf{u}$, with $\Omega(\mathbf{q})=v_{S} q$. Also, it should be noted that the dimensions of the generalized Raman tensor $R_{i j k}$ differ from the dimensions of $\gamma_{e}$ of Eq. (6) by a factor of (1/length).

In terms of unit polarization vectors, $\hat{e}^{(1)}, \hat{e}^{(2)}$ and $\hat{e}^{(Q)}$ for the three interacting modes, namely, the two optical modes and one phonon mode, respectively, relevant to the process being considered, one can also define a scalar effective Raman coupling constant for a bulk material, separately for the Stokes and the anti-Stokes interaction. For example, if 2 labels the anti-Stokes optical mode, for the anti-Stokes process one has the effective bulk coupling constant

$$
R^{(Q)}=\sum_{i j k} R_{i j k} \hat{e}_{i}^{(2) *} \hat{e}_{j}^{(1)} \hat{e}_{k}^{(Q)}
$$

For the special case of the long wavelength bulk longitudinal acoustic mode of wave vector $\mathbf{q}$, frequency $\omega_{m}$ and velocity $v_{S}$, described by Eqs. (10) and (11), one has

$$
4 \pi R_{i j k}=\mathrm{i} \gamma_{e} \delta_{i j} q_{k} ; \quad 4 \pi R^{(Q)}=\mathrm{i} \gamma_{e} q=\mathrm{i} \gamma_{e} \frac{\omega_{m}}{v_{S}},
$$

which follows on comparing Eqs. (14) and (15) with Eq. (8).

\section{INTERACTION BETWEEN ELECTROMAGNETIC FIELDS AND PHONONS IN A RESONATOR}

The coupled equations (10) and (12) are general enough to describe a variety of situations. Since we are interested here in cooling issues, we consider the case involving modes in resonators. We also specialize to the case of the anti-Stokes scattering. Let the electromagnetic field $\mathbf{E}$ consist of waves $\mathbf{E}_{1}$ and $\mathbf{E}_{2}$ at the frequency $\omega_{1}$ (called pump), and $\omega_{2}$ (anti-Stokes field). The frequencies $\omega_{1}$ and $\omega_{2}$ would be in the neighborhood of the resonator frequencies $\omega_{\mathrm{c} 1}$ and $\omega_{\mathrm{c} 2}$. Each resonator frequency would have certain line width. It is assumed that $\omega_{i}-\omega_{\mathrm{c} i}$ is within the line width of the mode. We can then write the field as

$$
\mathbf{E}_{i}(\mathbf{r}, t)=\vec{\phi}_{i} \mathcal{E}_{i}(t) \mathrm{e}^{-\mathrm{i} \omega_{i} t}+\text { c.c. } .
$$

In our formulation, we will use the familiar slowly varying amplitude approximation (SVAA) for finding the temporal variation of the field amplitudes $\mathcal{E}_{i}(t)$. Here $\vec{\phi}_{i}$ (with $\left.\nabla \cdot \vec{\phi}_{i}=0\right)$ is the spatial mode function for the electromagnetic mode in the resonator at the frequency $\omega_{\mathrm{c} i}$ such that $\nabla^{2} \vec{\phi}_{i}+\frac{\omega_{c i}^{2}}{c^{2}} \epsilon_{i} \vec{\phi}_{i}=0$. The amplitude $\mathcal{E}_{i}(t)$ is a slowly varying function of $t$. We use

$$
\begin{aligned}
\nabla^{2} \mathbf{E}_{i}-\frac{\epsilon_{i}}{c^{2}} \frac{\partial^{2} \mathbf{E}_{i}}{\partial t^{2}} & \cong\left(\nabla^{2} \vec{\phi}_{i}+\frac{\epsilon_{i}}{c^{2}} \omega_{i}^{2} \vec{\phi}_{i}\right) \mathcal{E}_{i} \mathrm{e}^{-\mathrm{i} \omega_{i} t}+ \\
& +2 \mathrm{i} \frac{\omega_{i} \epsilon_{i}}{c^{2}} \frac{\partial \mathcal{E}_{i}}{\partial t} \mathrm{e}^{-\mathrm{i} \omega_{i} t} \vec{\phi}_{i}+\text { c.c. }
\end{aligned}
$$

which on using $\omega_{i}^{2}-\omega_{\mathrm{c} i}^{2} \approx 2 \omega_{\mathrm{c} i}\left(\omega_{i}-\omega_{\mathrm{c} i}\right)=-2 \omega_{\mathrm{c} i} \Delta_{i}$ reduces to

$$
\begin{aligned}
& \nabla^{2} \mathbf{E}_{i}-\frac{\epsilon_{i}}{c^{2}} \frac{\partial^{2} \mathbf{E}_{i}}{\partial t^{2}} \\
\approx & 2 \vec{\phi}_{i} \frac{\epsilon_{i}}{c^{2}} \omega_{\mathrm{c} i}\left[\mathrm{i} \frac{\partial \mathcal{E}_{i}}{\partial t}-\Delta_{i} \mathcal{E}_{i}\right] \mathrm{e}^{-\mathrm{i} \omega_{i} t}+\text { c.c. }
\end{aligned}
$$

$\Delta_{i}=\omega_{\mathrm{c} i}-\omega_{i}$. Note that we have kept only the terms which are up to the first order in the time derivative or in the frequency difference $\Delta_{i}$. We next write the phonon mode as

$$
\mathbf{u}(\mathbf{r}, t)=\vec{\psi}(\mathbf{r}) u(t) \mathrm{e}^{-\mathrm{i} \omega_{m} t}+\text { c.c. },
$$

where, the mode function $\vec{\psi}(\mathbf{r})$ has the dimensions of the displacement field itself and $u(t)$ is the dimensionless amplitude of the mode. Note that phonons are interacting with a thermal bath, and depending on the strength of this interaction, the frequency spectrum of the amplitudes $u(t)$ for the given mode of frequency $\omega_{m}$ will represent the line width around the frequency $\omega_{m}$. When such phonons interact with the external optical fields via the three-wave interaction, this spectrum would be modified further. For longitudinal phonons curl $\vec{\psi}(\mathbf{r})=0$. Using the slowly varying amplitude approximation for the phonon mode amplitude also, the procedure that led to Eq. (18) now leads to

$$
-\nabla^{2} \mathbf{u}+\frac{\partial^{2} \mathbf{u}}{v_{S}^{2} \partial t^{2}} \approx 2 \frac{\vec{\psi}}{v_{S}^{2}} \omega_{m}\left[-\mathrm{i} \frac{\partial u}{\partial t}\right] \mathrm{e}^{-\mathrm{i} \omega_{m} t}+\text { c.c. . }
$$

Next we need to work out the interaction terms. Since we are considering the anti-Stokes process, $\omega_{1}+\omega_{m} \approx \omega_{2}$. This relation has to be satisfied within the line widths of all three modes $\omega_{\mathrm{c} 1}, \omega_{\mathrm{c} 2}$ and $\omega_{m}$ and hence all subsequent statements are to be understood within linewidths. Clearly a term like $\mathcal{E}_{1}^{*} \mathcal{E}_{2}$ on the right hand side of Eq.(10) will lead to the mode $\mathbf{u}$. Then the righthand side of Eq. (10) is to be approximated by

$$
\nabla E^{2}=\mathcal{E}_{1}^{*} \mathcal{E}_{2} \nabla\left(\vec{\phi}_{1}^{*} \cdot \vec{\phi}_{2}\right) \mathrm{e}^{\mathrm{i}\left(\omega_{1}-\omega_{2}\right) t}+\text { c.c. } .
$$

Similarly for deriving equation, say, for $\mathcal{E}_{2}$ from Eq. (12), the right hand side is to be approximated by

$$
\begin{aligned}
& -\frac{\gamma_{e}}{c^{2}} \frac{\partial^{2}}{\partial t^{2}}[(\nabla \cdot \mathbf{u}) \mathbf{E}] \\
\cong & \frac{\gamma_{e}}{c^{2}} \omega_{2}^{2}\left(\mathcal{E}_{1} u(\nabla \cdot \vec{\psi}) \vec{\phi}_{1}\right) \mathrm{e}^{-\mathrm{i}\left(\omega_{1}+\omega_{m}\right) t}+\text { c.c. } .
\end{aligned}
$$


On combining Eqs. (18)-(22) and carrying out all the simplifications, we get final equations for $\mathcal{E}_{1}, \mathcal{E}_{2}$ and $u$ :

$$
\begin{aligned}
\frac{\partial \mathcal{E}_{2}}{\partial t} & =-\kappa_{2} \mathcal{E}_{2}-\mathrm{i} \Delta_{2} \mathcal{E}_{2}-\mathrm{i} \frac{\chi_{2}}{\epsilon_{2}} \omega_{\mathrm{c} 2} u \mathcal{E}_{1} \mathrm{e}^{\mathrm{i} \delta t} \\
\frac{\partial \mathcal{E}_{1}}{\partial t} & =-\kappa_{1} \mathcal{E}_{1}-\mathrm{i} \Delta_{1} \mathcal{E}_{1}-\mathrm{i} \frac{\chi_{2}^{*}}{\epsilon_{1}} \omega_{\mathrm{c} 1} u^{*} \mathcal{E}_{2} \mathrm{e}^{-\mathrm{i} \delta t} \\
\frac{\partial u}{\partial t} & =-\Gamma u-\mathrm{i} \frac{\chi_{2}^{*} \mathcal{E}_{1}^{*} \mathcal{E}_{2} \mathrm{e}^{-\mathrm{i} \delta t}}{4 \pi \rho_{0} \omega_{m} \int \vec{\psi}^{*}(\mathbf{r}) \cdot \vec{\psi}(\mathbf{r}) d^{3} \mathbf{r}} \\
\delta & =\omega_{2}-\omega_{1}-\omega_{m} \\
\chi_{2} & =\frac{\gamma_{e}}{2} \int\left(\vec{\phi}_{2}^{*}(\mathbf{r}) \cdot \vec{\phi}_{1}(\mathbf{r})\right)(\nabla \cdot \vec{\psi}(\mathbf{r})) d^{3} \mathbf{r} .
\end{aligned}
$$

Here $\chi_{2}$ is the effective three mode coupling which depends on the overlap of all the modes. The integral in Eq. (27) is the traditional phase matching integral if plane waves are used for all the three modes in a bulk material. In writing Eqs. (23)-(27), we have added linewidth terms for all the modes. Note that $\gamma_{e}(\nabla \cdot \mathbf{u})$ is dimensionless and $\chi_{2}$ is dimensionless, the term $\mathcal{E}^{2}$ has dimensions of energy and $\rho_{0} v_{S}^{2}(\nabla \cdot \vec{\psi})^{2} u^{2}$ has dimensions of energy density, keeping in view that $u$ and $\nabla \cdot \vec{\psi}$ are dimensionless. The term $\rho_{0}$ in Eq. 25) can be eliminated by using the kinetic energy term $\frac{1}{2} \int \rho_{0}^{2}|\dot{\mathbf{u}}|^{2} d^{3} \mathbf{r}=\frac{1}{2} \rho_{0} \int|\mathbf{u}|^{2} \omega_{m}^{2} d^{3} \mathbf{r}=\rho_{0}|u|^{2} \omega_{m}^{2} \int \vec{\psi}^{*}(\mathbf{r})$. $\vec{\psi}(\mathbf{r}) d^{3} \mathbf{r}=\frac{\hbar \omega_{m}}{2}|u|^{2}$ and hence

$$
\rho_{0} \omega_{m}^{2} \int \vec{\psi}^{*}(\mathbf{r}) \cdot \vec{\psi}(\mathbf{r}) d^{3} \mathbf{r}=\frac{\hbar \omega_{m}}{2}
$$

We can also introduce a dimensionless quantity $\left(2 \pi \frac{\hbar \omega}{\epsilon}\right)|a|^{2}=|\mathcal{E}|^{2}$. In terms of the dimensionless quantities $a_{1}, a_{2}$ and $u$, the final equations are

$$
\begin{aligned}
& \frac{\partial a_{2}}{\partial t}=-\kappa_{2} a_{2}-\mathrm{i} \Delta_{2} a_{2}-\mathrm{i} \beta u a_{1} \mathrm{e}^{\mathrm{i} \delta t}, \\
& \frac{\partial a_{1}}{\partial t}=-\kappa_{1} a_{1}-\mathrm{i} \Delta_{1} a_{1}-\mathrm{i} \beta^{*} u^{*} a_{2} \mathrm{e}^{-\mathrm{i} \delta t}, \\
& \frac{\partial u}{\partial t}=-\Gamma u-\mathrm{i} \beta^{*} a_{1}^{*} a_{2} \mathrm{e}^{-\mathrm{i} \delta t},
\end{aligned}
$$

where the coupling constant $\beta$ is now given by

$$
\beta=\frac{\gamma_{e}}{2} \sqrt{\frac{\omega_{\mathrm{c} 2} \omega_{\mathrm{c} 1}}{\epsilon_{2} \epsilon_{1}}} \int\left[\vec{\phi}_{2}^{*}(\mathbf{r}) \cdot \vec{\phi}_{1}(\mathbf{r})\right](\nabla \cdot \vec{\psi}(\mathbf{r})) d^{3} \mathbf{r} .
$$

For a more general phonon mode described by the interaction density in Eq. (14), one can follow a similar procedure as detailed above for the longitudinal acoustic mode. Using the same symbols as in the equation (19), one can describe the general vibrational mode by its displacement field

$$
\mathbf{Q}(\mathbf{r}, t)=\vec{\psi}(\mathbf{r}) u(t) e^{-\mathrm{i} \omega_{m} t}+c . c,
$$

where $u(t)$ is again the slowly varying dimensionless amplitude of the phonon mode and $\vec{\psi}(\mathbf{r})$ is the corresponding mode function. Instead of introducing too many additional symbols, note that we are using the same symbols for mode functions and their amplitudes even for the general phonon mode. It requires a few words of caution. It should always be remembered that now $\nabla \times \vec{\psi}$ is not necessarily zero because it may not be representing a longitudinal phonon mode.

In such a case, one can show that we obtain the same set of final working equations (29) - (31), except that instead of the expression (32), the anti-Stokes coupling constant is now defined by

$$
\beta^{(Q)}=2 \pi \sqrt{\frac{\omega_{\mathrm{c} 2} \omega_{\mathrm{c} 1}}{\epsilon_{2} \epsilon_{1}}} \sum_{i j k} R_{i j k} \int \phi_{2 i}^{*}(\mathbf{r}) \phi_{1 j}(\mathbf{r}) \psi_{k}(\mathbf{r}) d^{3} \mathbf{r}
$$

It has to be noted that the resonator is being driven by a pump field of frequency $\omega_{1}$. Thus Eq.(29) is to be modified by adding the effect of the external field. We change $\kappa_{1} a_{1}$ term:

$$
\kappa_{1} a_{1} \rightarrow \kappa_{1}\left(a_{1}-\tilde{\mathcal{E}}_{1}\right)
$$

Here $\tilde{\mathcal{E}}_{1}$ is dimensionless and is determined by the resonator boundary conditions. The phonon mode is driven by thermal fluctuations, thus Eq. (31) is to be modified by converting it into a Langevin equation (see Eq. (43)). The Eq. (30) is also converted to Langevin equation by adding terms corresponding to the input vacuum field.

It is quite interesting to note that the basic equations (29) - (31) are quite generic in nature as these are applicable to a variety of situations. The details of a specific system enter through the coupling constant $\beta$ and the damping parameter $\Gamma^{\prime s}$ and $\kappa^{\prime} s$. The coupling constant Eq. (32) depends on the mode under consideration. We also note that in deriving equations (29) - (31) we have ignored the Stokes processes which are expected to be unimportant as we assume that there is no mode resonant with the Stokes frequency.

We also note that the coherent terms (i.e. terms without damping) in (29) - (31) can be obtained from the following effective Hamiltonian

$$
\mathcal{H}_{\mathrm{eff}}=\hbar \Delta_{1} a_{1}^{\dagger} a_{1}+\hbar \Delta_{2} a_{2}^{\dagger} a_{2}+\hbar\left(\beta^{*} b^{\dagger} a_{1}^{\dagger} a_{2} \mathrm{e}^{-\mathrm{i} \delta t}+\text { H.c. }\right),
$$

where we have used the second quantized notation $u \rightarrow b$, $u^{*} \rightarrow b^{\dagger}, a_{i}^{*} \rightarrow a_{i}^{\dagger}$. The operators $a_{i}^{\prime} s, a_{i}^{\dagger \prime} s, b$ and $b^{\dagger}$ satisfy Bosonic commutation relations. Under the assumption that the pump $a_{1}$ remains undepleted and is at resonance with the cavity mode $\left(\Delta_{1} \simeq 0\right), a_{1} \rightarrow \mathcal{E}_{1}$, Eq. (36) reduces to

$$
\mathcal{H}_{\text {eff }}=\hbar \Delta_{2} a_{2}^{\dagger} a_{2}+\hbar\left(\beta^{*} b^{\dagger} a_{2} \mathcal{E}_{1}^{*} \mathrm{e}^{-\mathrm{i} \delta t}+\text { H.c. }\right) .
$$

It should be borne in mind that the time dependence like $e^{-\mathrm{i} \omega_{2} t}$ and $e^{-\mathrm{i} \omega_{m} t}$ were separated out from $a_{2}$ and $b$ (Eq. (17) and Eq. (19)). If we work in a picture where such time dependences are kept, then instead of Eq. (37), we will have

$$
\mathcal{H}_{\text {eff }}=\hbar \omega_{\mathrm{c} 2} a_{2}^{\dagger} a_{2}+\hbar \omega_{m} b^{\dagger} b+\hbar\left(\beta^{*} b^{\dagger} a_{2} \mathcal{E}_{1}^{*} \mathrm{e}^{\mathrm{i} \omega_{1} t}+\text { H.c. }\right)
$$


It is important to keep track of the fast time dependences. Having derived Eq. (38) from first principles, we will use it's form to write the corresponding Hamiltonian for the case of two phonon modes.

\section{PHONON DISPERSION RELATIONS , CAVITY MODES AND DAMPING PARAMETERS}

Let us first consider the general problem of cooling phonon modes in a bulk material, which are characterized by the dispersion relation $\Omega(\mathbf{q})$, where $\mathbf{q}$ is the wave vector in a bulk material. Here the mode functions are plane waves. The phonon field is proportional to $\exp \{\mathrm{iq} \cdot \mathbf{r}-\mathrm{i} \Omega(\mathbf{q}) t\}$. We could have transverse phonons as in the case of Raman active optical phonons in solids, or longitudinal acoustic phonons as, say, in the case of Brillouin scattering. As already discussed earlier, the physical mechanism for cooling is the upconversion (antiStokes process) of light of frequency $\omega_{1}$ and wave vector $\mathbf{k}_{1}$. The upconversion is subject to the energy momentum conservation

$$
\Omega(\mathbf{q})+\omega_{1}\left(\mathbf{k}_{1}\right) \rightarrow \omega_{2}\left(\mathbf{k}_{2}\right), \quad \mathbf{q}+\mathbf{k}_{1}=\mathbf{k}_{2}
$$

In the upconversion process a thermal phonon of frequency $\Omega(\mathbf{q})$ is quickly removed. Further in order for the upconversion to be effective, we have to make sure that the process of downconversion (Stokes process) is avoided

$$
\omega_{1}\left(\mathbf{k}_{1}\right)-\Omega(\mathbf{q})=\omega_{s}\left(\mathbf{k}_{s}\right), \quad \mathbf{k}_{1}-\mathbf{q}=\mathbf{k}_{s}
$$

This is where the use of resonators and cavities becomes important. The form of the dispersion relation or the frequency spectrum of the phonon modes is also important. Several interesting forms of dispersion relations in simple geometries can be noted:

1. Bulk longitudinal sound waves - Brillouin mode: $\Omega(q)=v_{s} q$, where $v_{s}$ is the velocity of sound waves;

2. Bulk Raman active optical modes: $\Omega^{2}(q)=\Omega_{0}^{2}-$ $\alpha q^{2}$

3. Modes in confined geometries: it is well known that if the sample is confined to a length $d$ in any given direction, phonon modes propagating in that direction are discrete with spacing proportional to $\pi / d$. If the sample is confined in two of its dimensions, as in the case of phonons in an optical fiber, for a given discrete phonon mode in the transverse direction, the phonon frequency $\Omega(q)$ in the propagating direction is given by $\Omega^{2}(q) \approx \Omega_{0}^{2}+\alpha q^{2}$, where $\Omega_{0}$ for different discrete modes in the transverse direction is determined by the corresponding zero of the Bessel functions and thus it is inversely proportional to the radius of the fiber. In cases when $\Omega_{0} \neq 0$, the $q$ dependence of the frequency is not

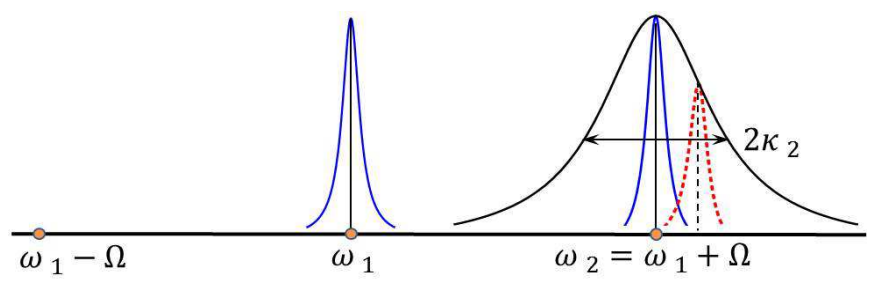

FIG. 1: Schematic representation of the anti-Stokes generation and the width of varies lines.

very significant and then the condition for momentum conservation in the direction of propagation is of a minor consequence. In a spherical resonator, which is confined in all the three directions, modes (both radial and azimuthal) are discrete.

One can arrange the resonator structure such that the upconversion process is resonant with the cavity mode whereas the downconversion process is non-resonant. Further one can choose the resonant cavity mode at $\omega_{2}$ to have large damping $\kappa_{2}$ so that the generated antiStokes field is quickly removed from the cavity. We assume that the width $\Gamma_{m}$ of the phonon mode is much smaller than the width of the cavity mode at the generated anti-Stokes frequency. We also assume that the pump laser at $\omega_{1}$ is monochromatic and that the line width of the corresponding resonator mode at $\omega_{1}$ is much smaller than $\kappa_{2}$. The situation is shown in the Fig 1 The anti-Stokes phonon could be exactly at the line center of the resonator mode at $\omega_{2}$. It depends on the energy conservation condition. The Figure 1 also implies that more than one phonon mode (see for example, the dotted curve) can be cooled simultaneously as long as these lie well within the width $2 \kappa_{2}$ of the generated anti-Stokes field; in other words if $\Omega_{1}$ and $\Omega_{2}$ are the center frequencies of two nearby sharp phonon modes, one should have $\left|\Omega_{1}-\Omega_{2}\right|<2 \kappa_{2}$. We consider this in detail in the next section.

\section{THEORETICAL DESCRIPTION OF BIMODAL COOLING}

Let us then first consider the case of two phonons modes in a resonator, one with frequency $\Omega_{1}$ and the other with frequency $\Omega_{2}$. Let the pump laser be at the frequency $\omega_{1}$. Then as required by the Fig 1 we assume that $\omega_{1}+\Omega_{i}(i=1,2)$ lie within the cavity line width $\kappa_{2}$ where the cavity is centered at the frequency $\omega_{\mathrm{c}}$. Under the assumption that the pump field remains un-depleted, the Hamiltonian for a system of two phonon modes $b_{1}$, $b_{2}$ and the cavity mode $a_{2}$ can be written as

$$
\begin{aligned}
H= & \hbar \omega_{\mathrm{c}} a_{2}^{\dagger} a_{2}+ \\
& +\sum_{i=1,2}\left\{\hbar \Omega_{i} b_{i}^{\dagger} b_{i}+\left(\beta_{i}^{*} b_{i}^{\dagger} a_{2} \mathcal{E}_{1}^{*} \mathrm{e}^{-\mathrm{i} \omega_{1} t}+\text { H.c. }\right)\right\},
\end{aligned}
$$


where we have replaced cavity mode $a_{1}$ by $\mathcal{E}_{1}$. The mode operators $a_{2}, b_{1}$ and $b_{2}$ satisfy Bosonic commutation relations. We assume that the cavity field is built up from the interaction of the pump field with thermal phonons. Let $\Omega_{0}$ be $\frac{\Omega_{1}+\Omega_{2}}{2}$ and $\Omega=\frac{\Omega_{1}-\Omega_{2}}{2}$, then we work in a picture obtained by making unitary transformation with

$$
\bar{H}=\hbar\left(\omega_{1}+\Omega_{0}\right) a_{2}^{\dagger} a_{2}+\hbar \Omega_{0}\left(b_{1}^{\dagger} b_{1}+b_{2}^{\dagger} b_{2}\right)
$$

to obtain an effective Hamiltonian

$$
\begin{aligned}
H_{\mathrm{eff}}= & \hbar \Omega\left(b_{1}^{\dagger} b_{1}-b_{2}^{\dagger} b_{2}\right)+\hbar \delta a_{2}^{\dagger} a_{2} \\
& +\sum_{i=1,2}\left(\beta_{i}^{*} b_{i}^{\dagger} a_{2} \mathcal{E}_{1}^{*} \mathrm{e}^{\mathrm{i} \delta t}+H . c .\right) \\
= & \hbar \Omega\left(b_{1}^{\dagger} b_{1}-b_{2}^{\dagger} b_{2}\right)+\hbar \delta a_{2}^{\dagger} a_{2} \\
& +\sum_{i=1,2}\left(G_{i}^{*} b_{i}^{\dagger} a_{2} \mathrm{e}^{\mathrm{i} \delta t}+H . c .\right), \\
\delta= & \omega_{\mathrm{c}}-\left(\omega_{1}+\Omega_{0}\right), \quad G_{i}=\beta_{i} \mathcal{E}_{1} .
\end{aligned}
$$

This is our working Hamiltonian which we use to obtain Heisenberg equations for $a_{2}, b_{i}$. We now need to introduce the dissipative terms which account for the leakage of phonons for the resonator and dissipation of the phonon modes and their then temperature. It is best then to write the quantum Langevin equations for the Heisenberg operators $a_{2}$ and $b_{i}$ :

$$
\begin{aligned}
& \dot{a}_{2}=-\mathrm{i} \delta a_{2}-\kappa_{2} a_{2}-\mathrm{i}\left(G_{1} b_{1}+G_{2} b_{2}\right)+f_{a_{2}}(t), \\
& \dot{b}_{1}=-\mathrm{i} \Omega b_{1}-\Gamma_{1} b_{1}-\mathrm{i} G_{1}^{*} a_{2}+f_{b_{1}}(t), \\
& \dot{b}_{2}=+\mathrm{i} \Omega b_{2}-\Gamma_{2} b_{2}-\mathrm{i} G_{2}^{*} a_{2}+f_{b_{2}}(t) .
\end{aligned}
$$

Here $\Gamma_{i}$ denotes the half width of the phonon mode $i$, $2 \kappa_{2}$ denotes the line width of the cavity mode.

Let us note here that the above set of equations are very generic, which can describe the coupling of any twomode system to a single optical field. In this sense, the resulting physics here in our case should be similar to the studies for other systems 12 15] involving these equations, including systems of oscillating membranes and trapped atoms.

In what follows, we would work in the limit $\Gamma_{i} \ll \kappa_{2}$; $\Omega_{i} \gg \kappa_{2}$. The force term $f_{j}(t)$ are the quantum Langevin forces. These are Gaussian forces with zero mean and with quantum correlations given by

$$
\begin{aligned}
\left\langle f_{a_{2}}^{\dagger}(t) f_{a_{2}}\left(t^{\prime}\right)\right\rangle & =0, \quad\left\langle f_{a_{2}}(t) f_{a_{2}}^{\dagger}\left(t^{\prime}\right)\right\rangle=2 \kappa_{2} \delta\left(t-t^{\prime}\right), \\
\left\langle f_{b_{i}}^{\dagger}(t) f_{b_{i}}\left(t^{\prime}\right)\right\rangle & =\frac{2 \Gamma_{i}}{\pi} \int_{-\infty}^{\infty} \bar{n}_{i}(\omega) \mathrm{e}^{-\mathrm{i} \omega\left(t-t^{\prime}\right)} d \omega, \\
\left\langle f_{b_{i}}(t) f_{b_{i}}^{\dagger}\left(t^{\prime}\right)\right\rangle & =\frac{2 \Gamma_{i}}{\pi} \int_{-\infty}^{\infty}\left(\bar{n}_{i}(\omega)+1\right) \mathrm{e}^{-\mathrm{i} \omega\left(t-t^{\prime}\right)} d \omega, \\
\left\langle f_{b_{1}}(t) f_{b_{2}}^{\dagger}\left(t^{\prime}\right)\right\rangle & =0 .
\end{aligned}
$$

A detailed derivation of the correlation functions in Eq. (44) can be found in books on quantum optics [1].
We first analyze the well known case [9] of the cooling of a single mode in the limit of large $\kappa_{2}$. We can then make the adiabatic approximation and write

$$
a_{2} \approx\left(\kappa_{2}+\mathrm{i} \delta\right)^{-1}\left(-\mathrm{i} G_{1}\right) b_{1},
$$

and then

$$
\frac{\partial b_{1}}{\partial t}+\mathrm{i} \Omega b_{1}+\left(\Gamma_{1}+\frac{\left|G_{1}\right|^{2}}{\kappa_{2}+\mathrm{i} \delta}\right) b_{1}=f_{b_{1}},
$$

i.e.

$$
\begin{aligned}
& \frac{\partial b_{1}}{\partial t}+\mathrm{i} \Omega_{1 \mathrm{eff}} b_{1}+\Gamma_{1 \mathrm{eff}} b_{1}=f_{b_{1}}, \\
& \Gamma_{1 \mathrm{eff}}=\left(\Gamma_{1}+\frac{\left|G_{1}\right|^{2} \kappa_{2}}{\kappa_{2}^{2}+\delta^{2}}\right), \\
& \Omega_{1 \mathrm{eff}}=\Omega_{1}-\frac{\delta\left|G_{1}\right|^{2}}{\kappa_{2}^{2}+\delta^{2}} .
\end{aligned}
$$

Thus the life time of the phonon mode goes down as $\Gamma_{1 \text { eff }}$ increases. The increase of $\Gamma_{1 \text { eff }}$ is subject to the assumption $\Gamma_{1 \mathrm{eff}}<\kappa_{2}$. The increase of $\Gamma$ leads to the cooling of the phonon mode, as the strength of the fluctuation $f_{b_{1}}$ is still determined by Equations in (44), i.e. by the damping parameter $\Gamma_{1}$. Note further that the fluctuation $f_{a_{2}}$ associated with the field mode does not contribute to the normally ordered moments as $\left\langle f_{a_{2}}^{\dagger}(t) f_{a_{2}}\left(t^{\prime}\right)\right\rangle=0$, which is the case as long as $\hbar \omega / k_{B} T \gg 1$.

\section{MODE-MODE COUPLING AND THE COLLECTIVE BEHAVIOR OF THE PHONON MODES}

We will now show that the adiabatic elimination of the cavity field results in mode-mode coupling. The resulting Langevin equations for phonon modes are given by

$$
\begin{gathered}
\frac{\partial b_{1}}{\partial t}+\left(\Gamma_{1}+\mathrm{i} \Omega\right) b_{1}=-\frac{\left|G_{1}\right|^{2} b_{1}+G_{1}^{*} G_{2} b_{2}}{\kappa_{2}+\mathrm{i} \delta}+f_{b_{1}}(t), \\
\frac{\partial b_{2}}{\partial t}+\left(\Gamma_{2}-\mathrm{i} \Omega\right) b_{2}=-\frac{\left|G_{2}\right|^{2} b_{2}+G_{2}^{*} G_{1} b_{1}}{\kappa_{2}+\mathrm{i} \delta}+f_{b_{2}}(t) .
\end{gathered}
$$

The mode-mode coupling can be thought of as the process

$$
\begin{aligned}
& \text { phonon } 1+\text { pump field } \rightarrow \text { anti-Stokes field } \\
\rightarrow & \text { phonon } 2+\text { pump field }
\end{aligned}
$$

It should be borne in mind that in the picture we work, all frequencies are matched due to Eq.(40). The actual generation of $b_{2}$ depends on the frequency mismatch factors $\Omega$ and $\delta$ as the solution of equations in (48) will obviously show.

Let us now consider the possibility of the collective behavior of the phonon modes. Let there be two identical 
phonon modes. This would be the case for two identical nano-mirrors placed at different nodes of an optical cavity (Similar to the situation of ref. 12]); We can then set $\Omega=0$. We drop $\Gamma_{i}$ assuming that the coupling field strength is such that $\Gamma_{\text {eff }} \sim \frac{\left|G_{1}\right|^{2} \kappa_{2}}{\kappa_{2}^{2}+\delta^{2}}$. Then equations in (48) reduce to

$$
\begin{aligned}
\frac{\partial b_{1}}{\partial t} & \approx-\frac{\left|G_{1}\right|^{2} b_{1}+G_{1}^{*} G_{2} b_{2}}{\kappa_{2}+\mathrm{i} \delta}+f_{b_{1}}(t), \\
\frac{\partial b_{2}}{\partial t} & \approx-\frac{\left|G_{2}\right|^{2} b_{2}+G_{2}^{*} G_{1} b_{1}}{\kappa_{2}+\mathrm{i} \delta}+f_{b_{2}}(t) .
\end{aligned}
$$

The two noise sources would also be identical. Further under the simplifying assumption $G_{1}=G_{2}=$ real

$$
\begin{aligned}
\frac{\partial b_{+}}{\partial t} & =-2 \frac{G_{1}^{2} b_{+}}{\kappa_{2}+\mathrm{i} \delta}+f_{+}(t), \\
\frac{\partial b_{-}}{\partial t} & =0+f_{-}(t)=0, \\
b_{ \pm} & =\frac{b_{1} \pm b_{2}}{\sqrt{2}}, \quad f_{ \pm}=\frac{f_{b_{1}} \pm f_{b_{2}}}{\sqrt{2}} .
\end{aligned}
$$

Under the conditions $\Gamma_{1}=\Gamma_{2}, \Omega=0$ used in deriving Eq. (50), $f_{-}$does not lead to nonvanishing contribution. Thus the collective mode $b_{-}$is sub-radiant whereas the mode $b_{+}$is super-radiant. The collective mode $b_{+}$has a decay rate which is twice than what it would be in the absence of any collective effects. Under these very special conditions there is enhanced cooling of only the collective mode $b_{+}$where $b_{-}$mode is unaffected. The collective effects are known in other contexts, for example in the cooling of two trapped ions [13] and in electro mechanical oscillators [12]. The collective modes in an ensemble of mechanical resonators trapped atoms in a Fabry-Perot resonator have been studied [14, 15].

\section{SPECTRUM OF THE GENERATED ANTI-STOKES FIELD AND COOLING OF THE PHONON MODES}

The spectrum of phonon fluctuations can be obtained by solving the quantum Langevin equations (43). Defining Fourier transformations via

$$
b(t)=\frac{1}{2 \pi} \int_{-\infty}^{\infty} b(\omega) \mathrm{e}^{-\mathrm{i} \omega t} d \omega,
$$

so that

$$
\begin{gathered}
\left\langle b^{\dagger}(t) b(t)\right\rangle=\frac{1}{2 \pi} \int_{-\infty}^{\infty} S_{b}(\omega) d \omega, \\
2 \pi S_{b}(\omega) \delta\left(\omega^{\prime}+\omega\right)=\left\langle b^{\dagger}\left(-\omega^{\prime}\right) b(\omega)\right\rangle .
\end{gathered}
$$

The quantity $S_{b}(\omega)$ gives the spectrum of phonon fluctuations. A reduction in the value of $\left\langle b^{\dagger} b\right\rangle$ when $G^{\prime s} \neq 0$, would represent cooling. Using the properties of the

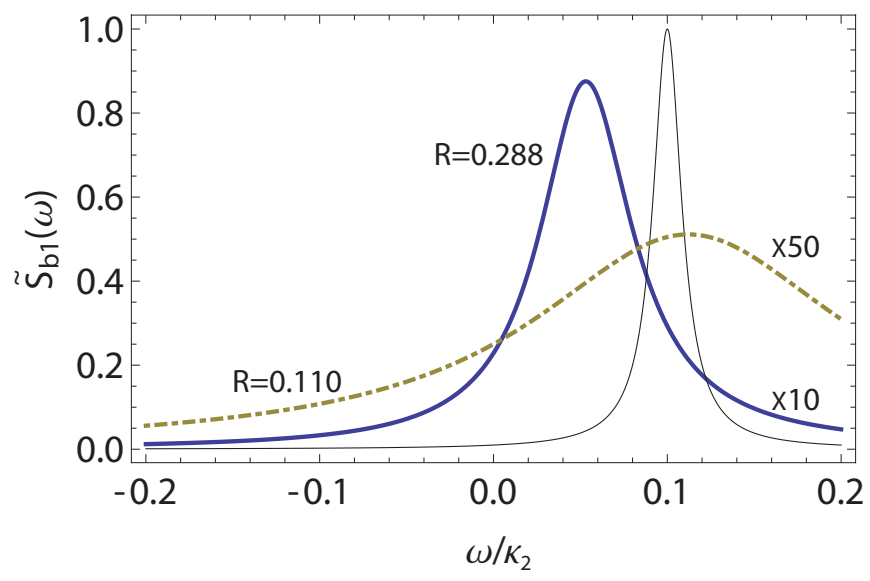

FIG. 2: The spectrum $\tilde{S}_{b_{1}}(\omega)=\frac{\Gamma_{1} S_{b_{1}}(\omega)}{2 n_{1}}$ as a function of $\omega / \kappa_{2}$ for $\frac{\Gamma_{1}}{\kappa_{2}}=\frac{\Gamma_{1}}{\kappa_{2}}=0.01 ; \frac{\Omega}{\kappa_{2}}=0.1$ and $\frac{G_{1}}{\kappa_{2}}=0.3, G_{2}=0$ (green curve), $\frac{G_{1}}{\kappa_{2}}=0.3, \frac{G_{2}}{\kappa_{2}}=0.5$ (blue curve). The black curve represents spectrum when both $G_{1}=G_{2}=0$. The parameter $R$ (Eq. [56) gives the extent of cooling.

Langevin forces we find the final results for the spectra of the two phonon modes

$$
\begin{aligned}
S_{b_{1}}(\omega)= & \frac{2 \Gamma_{1} n_{1}(\omega)\left|\left(\mathrm{i} \delta-\mathrm{i} \omega+\kappa_{2}\right)+\frac{\left|G_{2}\right|^{2}}{-\mathrm{i} \Omega-\mathrm{i} \omega+\Gamma_{2}}\right|^{2}}{|d(\omega)|^{2}\left[(\Omega-\omega)^{2}+\Gamma_{1}^{2}\right]} \\
& +\frac{2 \Gamma_{2} n_{2}(\omega)\left|\frac{G_{1} G_{2}}{-\mathrm{i} \Omega-\mathrm{i} \omega+\Gamma_{2}}\right|^{2}}{|d(\omega)|^{2}\left[(\Omega-\omega)^{2}+\Gamma_{1}^{2}\right]} \\
& \stackrel{G_{1}, G_{2}=0 \frac{2 \Gamma_{1} n_{1}(\omega)}{\left[(\Omega-\omega)^{2}+\Gamma_{1}^{2}\right]}}{[(\Omega)}
\end{aligned}
$$

$$
\begin{aligned}
S_{b_{2}}(\omega)= & \frac{2 \Gamma_{2} n_{2}(\omega)\left|\left(\mathrm{i} \delta-\mathrm{i} \omega+\kappa_{2}\right)+\frac{\left|G_{1}\right|^{2}}{-\mathrm{i} \Omega-\mathrm{i} \omega+\Gamma_{1}}\right|^{2}}{|d(\omega)|^{2}\left[(\Omega+\omega)^{2}+\Gamma_{2}^{2}\right]} \\
& +\frac{2 \Gamma_{1} n_{1}(\omega)\left|\frac{G_{1} G_{2}}{\mathrm{i} \Omega-\mathrm{i} \omega+\Gamma_{1}}\right|^{2}}{|d(\omega)|^{2}\left[(\Omega+\omega)^{2}+\Gamma_{2}^{2}\right]} \\
& \stackrel{G_{1}, G_{2}=0}{\longrightarrow} \frac{2 \Gamma_{2} n_{2}(\omega)}{\left[(\Omega+\omega)^{2}+\Gamma_{1}^{2}\right]}
\end{aligned}
$$

where

$d(\omega)=\left(\mathrm{i} \delta-\mathrm{i} \omega+\kappa_{2}\right)+\frac{\left|G_{1}\right|^{2}}{\mathrm{i} \Omega-\mathrm{i} \omega+\Gamma_{1}}+\frac{\left|G_{2}\right|^{2}}{-\mathrm{i} \Omega-\mathrm{i} \omega+\Gamma_{2}}$.

The spectrum of fluctuations, given by equations in (54), should be compared with the unperturbed spectrum $\left(G_{1}=G_{2}=0\right)$. For typical parameters we show the results in Figs. 22 and 3. The parameters $\kappa_{2}, \Gamma^{\prime} s$ and $G^{\prime} s$ that we choose, are similar to the case of a single 


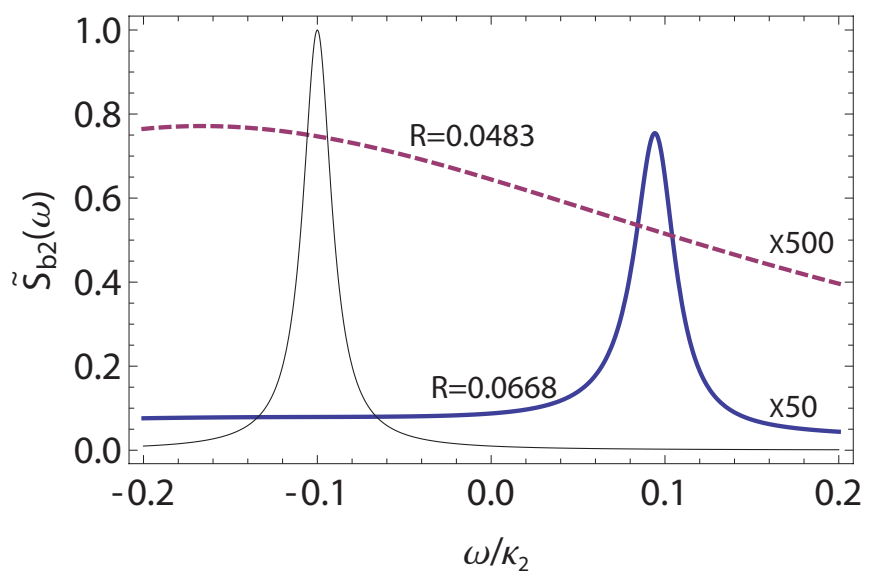

FIG. 3: Same as in Fig, 2, except for the spectrum $\tilde{S}_{b_{2}}(\omega)=$ $\frac{\Gamma_{2} S_{b_{2}}(\omega)}{2 n_{2}}$ of the $b$ mode. The pink graph is for $G_{1}=0, \frac{G_{2}}{\kappa_{2}}=$

phonon mode 4]. Although the coupling of the three modes makes $d(\omega)$ cubic function of $\omega$, the spectrum in the vicinity of $\omega=\Omega(\omega=-\Omega)$ exhibits a single resonance for $\delta=0, \Gamma \ll \kappa$. The Figs. 2 and 3 show the cooling of both modes. The mode-mode coupling leads to heating, however overall both modes get cooled. The ratio $R$ gives the ratio of $\left\langle b^{\dagger}(t) b(t)\right\rangle$ in presence of the coupling to it's value in the absence of coupling i.e. $R$ for example in Fig. 2 is

$$
R=\frac{\left\langle b_{1}^{\dagger}(t) b_{1}(t)\right\rangle}{\bar{n}_{1}} .
$$

It should be emphasized that the cooling parameter $R$ introduced here should not be confused with the effective bulk Raman coupling constant $R^{(Q)}$ introduced in Eq. (15). For Fig,2 for the values of $G_{1}$ and $G_{2}$ used, $R=0.110$ if $G_{2}=0 ; R=0.288$ if $G_{2} \neq 0$. The parameters $G_{1}$ and $G_{2}$ [Eq. (42)] depend on the phase matching parameter $\beta$ and the power of the Stokes field. Clearly if we increase the power of the Stokes laser i.e. if we increase $G$, then $R$ goes down. We note that the parameter $R$ also gives the ratio of the temperature of the cooled phonon mode to its initial temperature. This is because the factor $\left(e^{\hbar \omega / k_{\mathrm{B}} T}-1\right)^{-1} \approx \frac{k_{\mathrm{B}} T}{\hbar \omega}$ if $\frac{k_{\mathrm{B}} T}{\hbar \omega} \ll 1$. For phonon frequency, say in the range, $100 \mathrm{MHz}$ [4], the parameter $\frac{k_{\mathrm{B}} T}{\hbar \omega} \ll 1$ even at a temperature like $1 \mathrm{~K}$. by

The spectrum of the generated anti-Stokes field is given

$S_{a}(\omega)=\frac{2 \Gamma_{1} n_{1}(\omega)\left|\frac{G_{1}}{\mathrm{i} \Omega-\mathrm{i} \omega+\Gamma_{1}}\right|^{2}+2 \Gamma_{2} n_{2}(\omega)\left|\frac{G_{2}}{-\mathrm{i} \Omega-\mathrm{i} \omega+\Gamma_{2}}\right|^{2}}{|d(\omega)|^{2}}$,

which is shown in Fig, 4 . The figure also shows the effect of mode mixing. The mode mixing arises from the modification of the denominator in Eq. (57). The numerator in Eq. (57) represents the conversion of phonons into

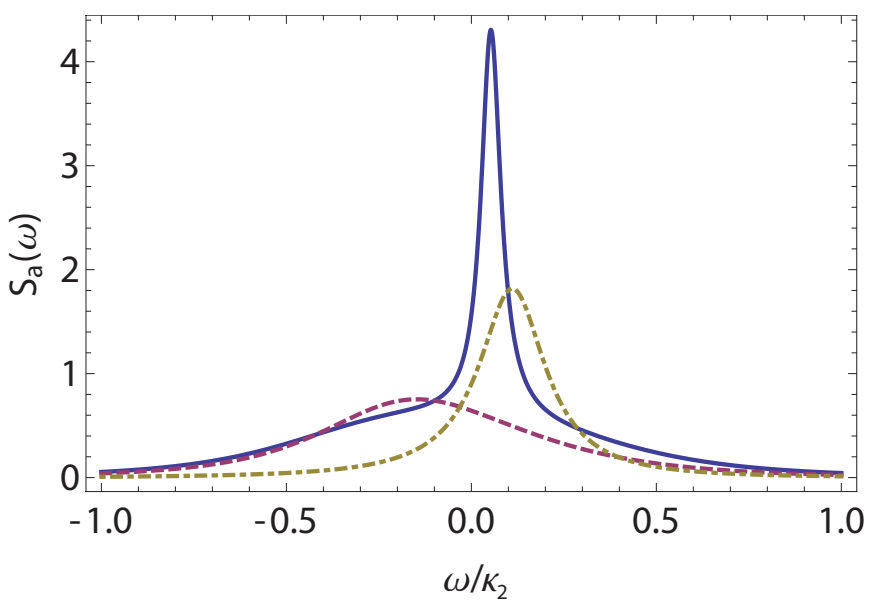

FIG. 4: Spectrum of the generated anti-Stokes for $\frac{G_{1}}{\kappa_{2}}=0.3$, $G_{2}=0$ (green); $G_{1}=0, \frac{G_{2}}{\kappa_{2}}=0.5$ (pink); $\frac{G_{1}}{\kappa_{2}}=0.3, \frac{G_{2}}{\kappa_{2}}=0.5$ (blue).

anti-Stokes in the lowest order of the coupling $G_{1}$ and $G_{2}$. Note that the spectrum of the generated antiStokes radiation has signatures of the phonon spectrum. The spectrum of the generated radiation can be studied by examining the output field at frequency $\omega_{2}$. Note that the anti-Stokes photons leak out of the cavity and the phonon modes are constantly interacting with the thermal bath, the conservation of photon and phonon numbers in the cavity does not hold.

If we use the actual numerical parameters of the experiment on the single- mode cooling of the acoustic phonon modes done by Bahl et al. [4], we can also examine the possibility of bimodal phonon cooling in such a system. In their experiment, $\kappa_{2}$ was of the order of $5 \mathrm{MHz}$, and they had observed cooling of the surface acoustic phonon mode of frequency $95 \mathrm{MHz}$, with their intrinsic line width of the order of only $8 \mathrm{kHz}$ at the ambient temperature of $294 \mathrm{~K}$. Thus, if one can find another similar phonon mode in the system with its frequency within a few $\mathrm{MHz}$ away from the $95 \mathrm{MHz}$ mode, say in the range of 90 to $100 \mathrm{MHz}$, which also has an appreciable coupling $\beta$, defined by Eq. (32), we should be able to observe simultaneous cooling of two such modes in the same experimental system. Otherwise, one has to search for another appropriate phonon system to observe this effect. Note that in the experimental system discussed above, if there are more than two phonon modes near the $95 \mathrm{MHz}$ mode within $2 \kappa_{2}$ range, each one with appreciable coupling, all those modes are expected to be cooled simultaneously.

In conclusion, we have presented a general theory of cooling of phonon modes via their interactions with suitable optical fields. The theory is formulated in terms of the spatial mode functions for the relevant interacting modes, in any given geometry, allowing it to be applicable to a variety of systems with diverse phonon dispersion relations and frequency spectra. In particular, we show 
the cooling of two phonon modes at the same time if the line width of each of the phonon modes is very small compared to the decay rate $2 \kappa_{2}$ of the resonant anti-Stokes cavity mode and their frequency difference is smaller than the line width $2 \kappa_{2}$. In any experimental set-up similar to that used by Bahl et al. [4], if one can find two nearby sharp phonon modes satisfying the above conditions, one should be able to observe the bimodal cooling described in this paper. We also find in this paper the possibility of collectively enhanced cooling of phonon modes under suitable conditions. However, further studies are needed to identify such a system with required phonon mode characteristics. A generalization of the present work to four wave parametric interactions (for example Hamiltonians which are quadratic in $b_{i}^{\prime}{ }^{s}$ ) would also be interesting.

One of us (SSJ) acknowledges the hospitality of the Oklahoma State University, while this work was done.
[1] M. Aspelmeyer, T. J. Kippenberg, and F. Marquardt, arXiv: 1303.0733. M. Aspelmeyer, P. Meystre, and K. Schwab, Physics Today 65, 29 (2012).

[2] Young-Shin Park and Hailin Wang, Nature Physics 5, 489 (2009); J. D. Teufel, et al., Nature 475, 359 (2011).

[3] G. S. Agarwal, Quantum Optics, (Cambridge University Press, NewYork, 2012), Chap. 20.

[4] Gaurav Bahl, Matthew Tomes, Florian Marquardt, and Tal Carmon, Nat. Phys. 8, 203 (2012).

[5] R. W. Boyd, Nonlinear Optics, (Academic Press, NewYork, 1992), Chap. 8.

[6] Aydin Yeniay, Journal of lightwave technology 20, 1425 (2002).

[7] P. Dainese, P. St. J. Russell, G. S. Wiederhecker, N. Joly, H. L. Fragnito, V. Laude, and A. Khelif, Opt. Express 14, 4141 (2006).

[8] Peter T. Rakich, Charles Reinke, Ryan Camacho, Paul Davids, and Zheng Wang, Phys. Rev. X 2, 011008 (2012).
[9] Matthew Tomes, Florian Marquardt, Gaurav Bahl, and Tal Carmon, Phys. Rev. A 84, 063806 (2011).

[10] J. D. Jackson, Classical Electrodynamics, (John Wiley \& Sons, Inc., 1998), Eq. (4.94).

[11] See ref [3], Eqs. (9.86), p. 212; M. O. Scully and M. S. Zubairy, Quantum Optics, (Cambridge University Press, NewYork, 1997), Sec 9.1, Eqs. (9.1.15), (9.1.24), (9.1.27).

[12] Francesco Massel, Sung Un Cho, Juha-Matti Pirkkalainen, Pertti J. Hakonen, Tero T. Heikkilä, and Mika A. Sillanpä̈̈, Nat. Comm. 3, 987 (2012).

[13] B. E. King, C. S. Wood, C. J. Myatt, Q. A. Turchette, D. Leibfried, W. M. Itano, C. Monroe, and D. J. Wineland, Phys. Rev. Lett. 81, 1525 (1998).

[14] André Xuereb, Claudiu Genes, and Aurélien Dantan, Phys. Rev. Lett. 109, 223601 (2012).

[15] Monika H. Schleier-Smith et al., Phys. Rev. Lett. 107, 143005 (2011). 